\title{
STABILIZING THE OPERATION OF INDUSTRIAL PROCESSES USING DATA DRIVEN TECHNIQUES
}

\author{
M. A. A. Shoukat Choudhury ${ }^{* 1,}$, Ian Alleyne ${ }^{2}$ \\ ${ }^{1}$ Department of Chemical Engineering, Bangladesh University of Engineering and Technology, Dhaka-1000, Bangladesh \\ ${ }^{2}$ Department of Chemical and Materials Engineering, University of Alberta, Edmonton AB, Canada
}

Received 4 August 2009; received in revised form 5 October 2009

\begin{abstract}
Poor performance of a control loop is usually caused by poor controller tuning, presence of disturbances, control loop interactions and/or loop nonlinearities. The presence of nonlinearities in control loops is one of the main reasons for poor performance of a linear controller designed based on linear control theory. In a control loop, nonlinearities may appear either in the control instruments such as valves and positioners or in the process. Among the control valve nonlinearities stiction, deadband, deadzone, hysteresis and saturation are most common. A nonlinear system often produces a non-Gaussian and nonlinear time series. The test of Gaussianity or nonlinearity of a control loop variable serves as a useful diagnostic aid towards diagnosing the causes of poor performance of a control loop. Two indices, the Non-Gaussianity Index (NGI) and the Non-Linearity Index $(N L I)$, developed in [1] are used to detect the possible presence of nonlinearity in the loop. These indices together with specific patterns in the process output ( $p v)$ vs. the controller output $(o p)$ plot can be conveniently used to diagnose the causes of poor control loop performance thus ensuring smooth operation of the plant. The method has been successfully applied to many industrial data sets. One of the interesting case studies is presented in this paper. The results of the analysis were confirmed and the results after the troubleshooting was performed are also presented.
\end{abstract}

Keywords: Nonlinearities, stiction, performance monitoring, nonGaussianity, process industries, control valves

DOI:10.3329/cerb.v13i1.2995

\section{Introduction}

In the process industry, control and automation is increasingly the route to achieve various objectives such as maintaining world class quality of the product, reducing operating and maintenance cost, enhancing operators' safety, meeting environmental and occupational health regulations, optimizing resource manage-

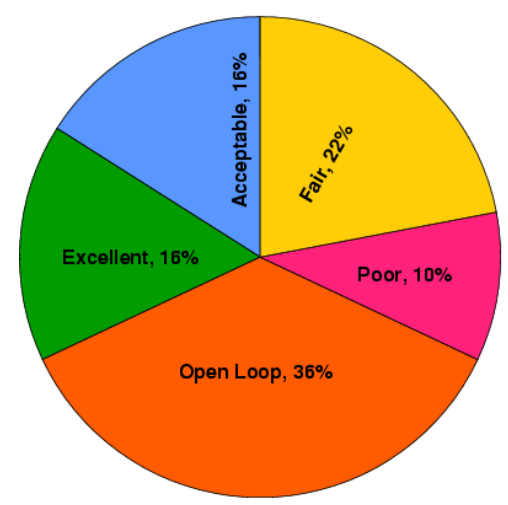

Figure 1: Global multi-industry performance demographics

\footnotetext{
*Corresponding author Email: shoukat@che.buet.ac.bd
}

ment and increasing profitability. One manifestation of this increased automation is an increasing number of controllers and control loops in process industries. A process control plant may have anywhere from a few control loops to several thousands, depending on the complexity of the plant from the perspective of control. The performance assessment and monitoring of the performance of these control loops are crucial to the achievement of the desired objectives. Performance demographics of 26,000 PID controllers collected over a period of two years and across a large range of continuous process industries have been discussed by [2]. The results are reproduced in Figure 1.

Each type of control loop (flow, pressure, level, temperature, etc.) was classified into one of the five categories - excellent, acceptable, fair, poor and open loop - based on a combined algorithm of minimum variance benchmark and an oscillation metric. The classifications were further refined through extensive validation and feedback from industry to reflect controller performance relative to practical expectations for each measurement type. It is evident from Figure 1 that only a third of the loops are performing well or in an acceptable fashion. The other two-thirds have significant opportunity for improvement. The key to (C) Bangladesh Uni. of Engg. E Tech. 
improving their performance is to diagnose the causes behind their poor performance.

\section{Problem Description}

Figure 2 shows a typical control loop under feedback configuration. The objective of this control loop may be either set point tracking or disturbance rejection. In practice, data for only three measurements for each control loop are available. They are set point (SP), controlled variable (PV), and controller output (OP or sometimes also termed CO or MV) signals. The measurement of actual control valve stem position is seldom available. Many authors [3-6] have discussed methods, for example, minimum variance benchmark and settling time benchmark, to estimate controller performance or loop performance from routine operating data. In many cases, this information is also available from plant engineers or operators, who are dissatisfied with the poorly performing loops. The challenge here is to identify the root cause of a poorly performing loop from routine operating (SP, PV and OP) data.

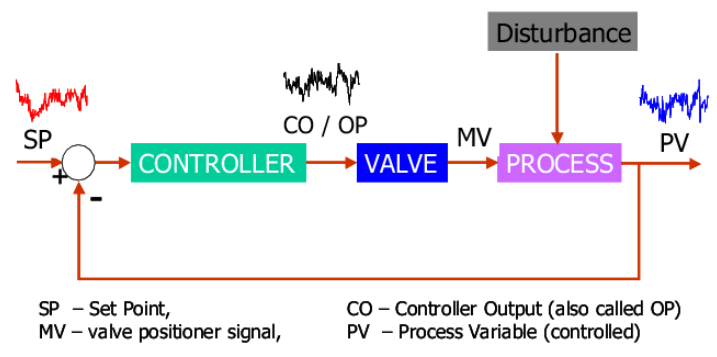

Figure 2: Global multi-industry performance demographics

\section{Causes of Poor Performance}

Jelali [7] has provided a comprehensive overview of the control performance assessment technology and their limitations. Poor performance of a control loop is usually due to the following:

a. Poor Controller Tuning: Often times the controllers are left with the default tuning settings from commissioning of the plant, even though there may be a lot of changes in the process operation or process modification over the years which demands retuning of the controller parameters.

b. Equipment Failures: Failure or malfunction of sensors or actuators may result in poor control performance. In most process industries process control engineers have limited time to devote to individual control loops and maintain them properly.

c. Poor Process Design: There should be a proper co-ordination between process design engineers and control engineers during the design phase of a chemical plant in order to ensure better controllability. It is hard to achieve good control performance on an improperly designed process. For example, the current practice in process design tries to minimize the liquid hold-up at the bottom of a distillation column, however such a design offers little surge capacity to filter out or reject disturbances. A nice case study in this area can be found at [8]. The use of reduced inventory and small buffer capacity, recycle streams and tight energy integration contributes to strong loop interactions, plantwide oscillation propagations and thereby leads to poor controller performance.

d. Loop Interactions: Inadequate compensation for interacting loops, improper loop pairing and competing controllers may result in poor control performance.

e. Not using Derivative Component in the PID controller: In most process applications, the derivative components of PID controllers are left out because of the inability or lack of expertise in designing a good filter required to use the derivative (D) part of the controller. Birgitta [9] and Adeleye [10] have showed that with the use of either first or second order filters, a PID controller can significantly improve the control performance over a PI controller. Also, for processes having significant time delays, substantial improvement in closed-loop performance can be achieved if PID controllers are used instead of PI controllers.

f. Presence of Nonlinearities: Loop nonlinearities can cause poor performance because controllers are usually designed based on linear control theory assuming everything in the loop is locally linear. The nonlinearities in a loop may arise due to the presence of actuator or valve nonlinearities and/or nonlinear nature of the process itself.

\section{Methodology for Poor Performance Diagnosis}

Poor control performance can be attributed to specific causes related to either linearity or nonlinearity. The linearity related causes include tightly tuned controllers, linear oscillatory disturbances and loop interactions. The nonlinearity related causes include valve nonlinearities or process nonlinearities.

\subsection{Detection of loop nonlinearity}

A control loop containing valve nonlinearities often produces non-Gaussian (e.g., a signal with asym- 
metric distribution) and nonlinear time series, namely process output (PV) and controller output (OP) data. Higher order statistics based nonlinearity assessment can be used as a diagnostic tool for troubleshooting of hardware faults that may be present in the control loop $[11,12]$. The test of Gaussianity and nonlinearity of the control error signal (SP-PV) is a useful diagnostic aid for determining the poor performance of a control loop. The test described in [1] uses the sensitivity of the normalized bispectrum or bicoherence to detect the presence of nonlinear interactions in the signal. A distinctive characteristic of a nonlinear time series is the presence of phase coupling such that the phase of one frequency component is determined by the phases of others. Phase coupling leads to higher order spectral features that can be detected in the bicoherence of a signal. The nonlinearity test applied here uses bicoherence to assess the nonlinearity. Bicoherence is defined as:

$$
\operatorname{bic}^{2}\left(f_{1}, f_{2}\right) \triangleq \frac{\left|B\left(f_{1}, f_{2}\right)\right|^{2}}{E\left[\left|X\left(f_{1}\right) X\left(f_{2}\right)\right|^{2}\right] E\left[\left|X\left(f_{1}+f_{2}\right)\right|^{2}\right]}(1)
$$

where $B\left(f_{1}, f_{2}\right)$ is the bispectrum at frequencies $\left(f_{1}, f_{2}\right)$ and is given by:

$$
B\left(f_{1}, f_{2}\right) \triangleq E\left[X\left(f_{1}\right) X\left(f_{2}\right) X^{*}\left(f_{1}+f_{2}\right)\right]
$$

$X\left(f_{1}\right)$ is the discrete Fourier transform of the time series $x(k)$ at the frequency $f_{1}, X^{*}\left(f_{1}\right)$ is the complex conjugate and $E$ is the expectation operator. A key feature of the bispectrum is that it has a non-zero value if there is a significant phase coupling in the signal $x$ between frequency components at $f_{1}$ and $f_{2}$. The bicoherence gives the same information but is normalized as a value between 0 and 1 .

In Choudhury et al. [12], two indices - the Non-Gaussianity Index $(N G I)$ and the NonLinearity Index $(N L I)-$ have been defined as:

$$
\begin{aligned}
& N G I \triangleq \overline{b \hat{i}^{2}}-\overline{b i c^{2}}{ }_{c r i t} \\
& \left.N L I \triangleq \mid b \hat{i c_{\text {max }}^{2}}-\overline{\left(b \hat{i c}^{2}\right.}+2 \sigma_{b \hat{i c}^{2}}\right) \mid
\end{aligned}
$$

The logic flow diagram in Figure 3 demonstrates the application of HOS based techniques in distinguishing linear and nonlinear causes of poor control performance.

First, the control error signal is examined by the HOS based nonlinearity test. If nonlinearity is not detected, the focus of the diagnosis should be on controller tuning or on the possible presence of an external linear oscillatory disturbance. If both NGI and NLI are greater than their statistical critical values, then the signal is described as non-Gaussian and nonlinear. After a loop is identified as nonlinear, the causes of nonlinearity should be diagnosed. With the assumption of a locally linear process, it can be concluded that the valve is the most likely responsible element for the loop nonlinearity. The next problem is to diagnose whether this valve nonlinearity is due to stiction or some other problems. An elliptical pattern in the pv-op plot is an indication of valve stiction.

\section{Quantification of Valve Stiction}

Strictly speaking, all valves are sticky to some extent. A detection and diagnosis algorithm can identify stiction in a large number of control valves. Some of them may be sticky by an acceptably small amount for the current application in hand while others may suffer from severe stiction and need immediate maintenance of the valve. Therefore, it is important to be able to quantify stiction so that a list of sticky valves in order of their maintenance priority can be prepared. It is well known [13-16] that the presence of stiction in control valve in a control loop produces limit cycles in the controlled variable $(p v)$ and the controller output,op. For such a case, if $p v$ is plotted against $o p$, it produces cyclic patterns in the resulting $p v-o p$ plot.

This is a level control loop in a power plant which controls the level of condenser located at the outlet of a turbine by manipulating the flow rate of the liquid condensate.

Figure 4 a shows the time trends of the $s p, p v$, and $o p$ data. The values of NGI and NLI calculated for this loop were found to be 0.32 and 0.83 . These indices clearly indicate that nonlinearity was present in this loop. Figure $4 \mathrm{~b}$ shows a typical $p v-o p$ plot for a sticky loop. A large number of such plots can be found in Choudhury et al. [17], where stiction models were used in a closed loop SISO system to produce data for these plots. An ellipse can be fitted to such a $p v-o p$ plot (shown in the figure). The stiction can be quantified or estimated as the maximum width of the cycles of the $p v-o p$ plot at the direction of $o p$. The quantified stiction is termed as 'apparent stiction' because the actual amount of stiction to be obtained from the $m v-o p$ plot may differ from the estimated quantity because of the effect of loop dynamics on the controlled variable, $p v$, in particular, the effect of the controller to compensate or fight stiction.

\section{Stabilization of the Operation of a Plastic Man- ufacturing Process}

The methodology described above was used to stabilize one unit of a polymerisation unit in a plastic plant. The idea was to identify and correct the root cause which was destabilizing this particular unit. This particular polymerisation unit produces 55000 ton/year of 30 different grade polymers. The melting indices of these polymers vary from 0.3 to 1000 


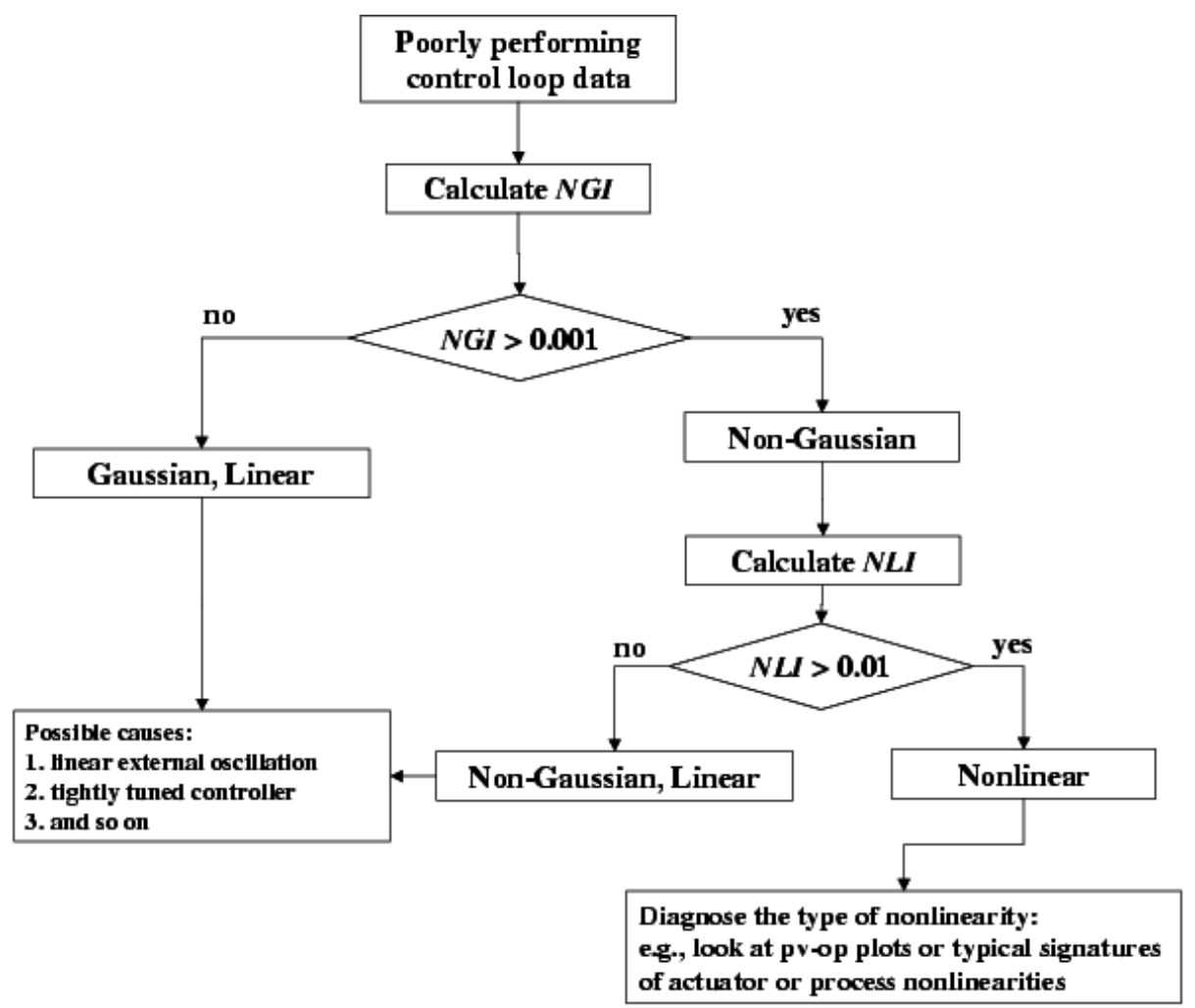

Figure 3: Decision flow diagram for poor performance diagnosis
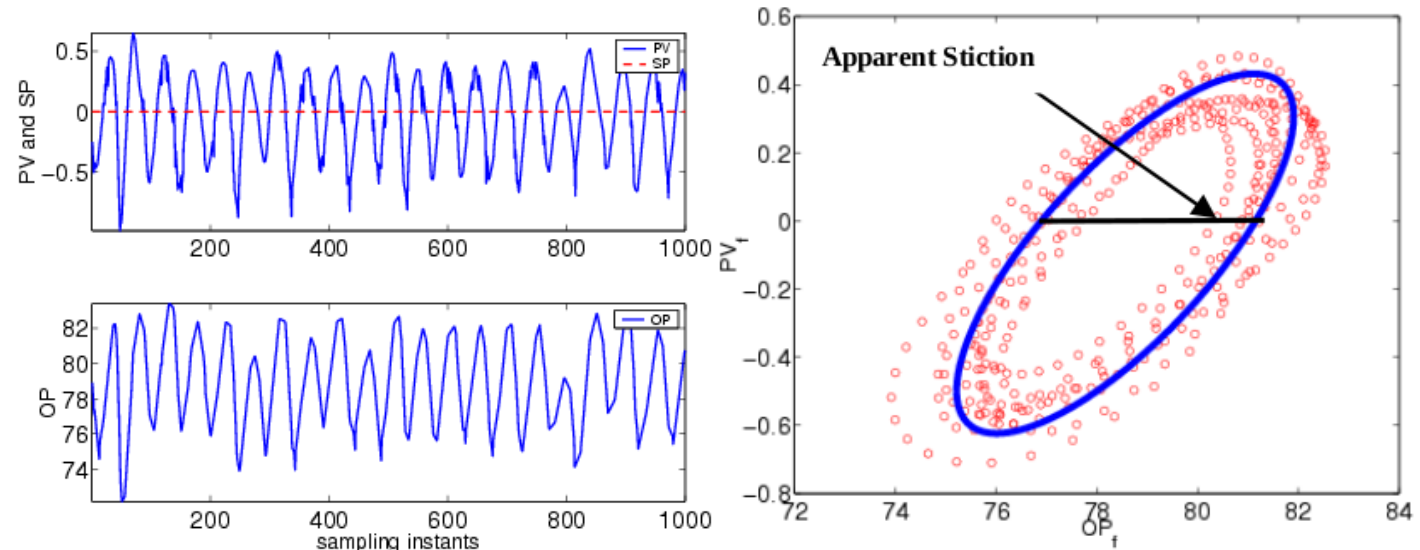

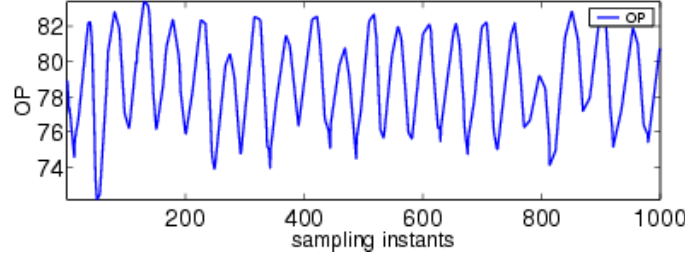

(a)

(b)

Figure 4: (a) Time trends of a level loop containing a sticky valve; (b) The $p v-o p$ plot for a level loop containing a sticky valve 


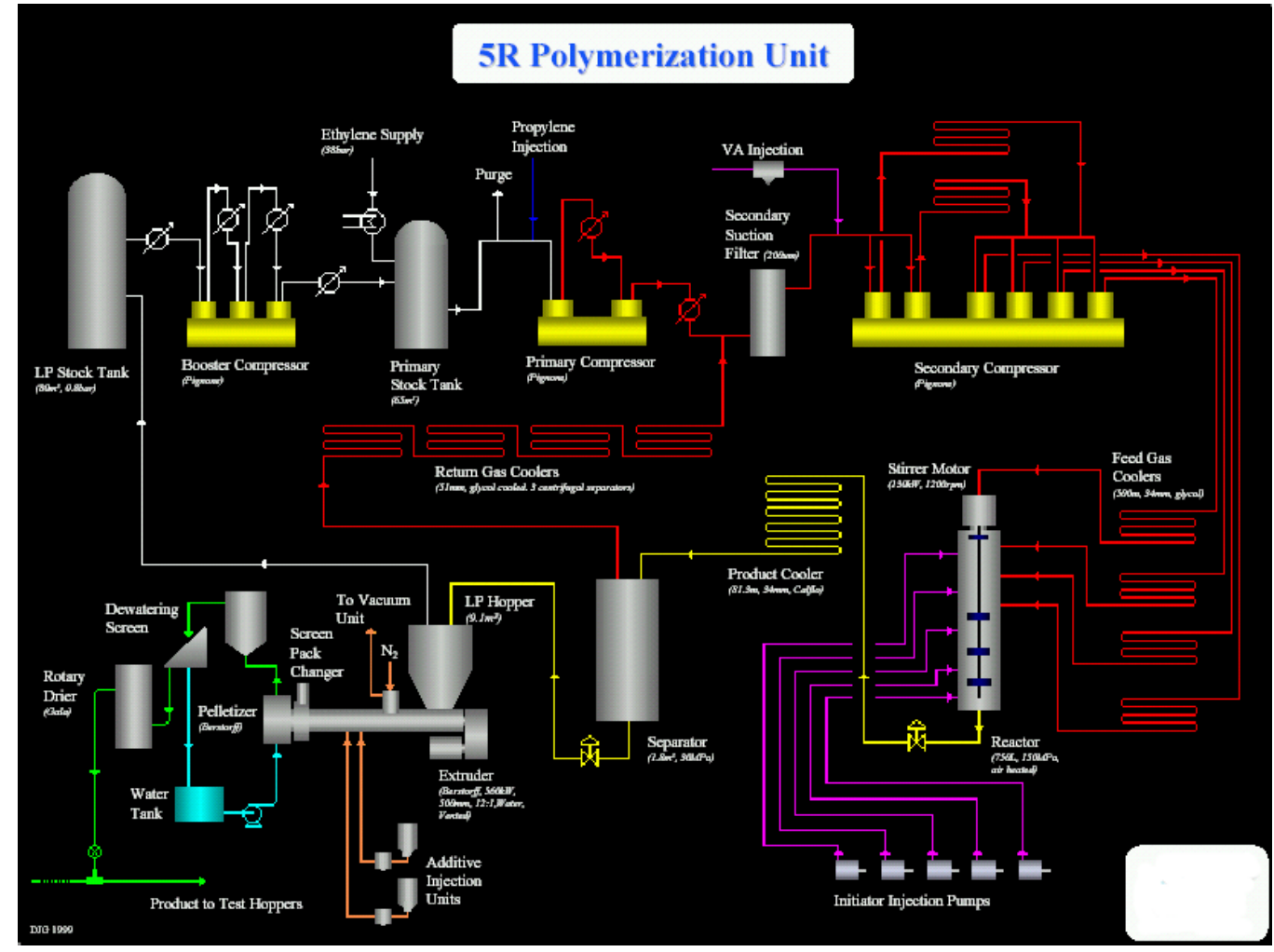

Figure 5: Process flow diagram of the polymerization unit

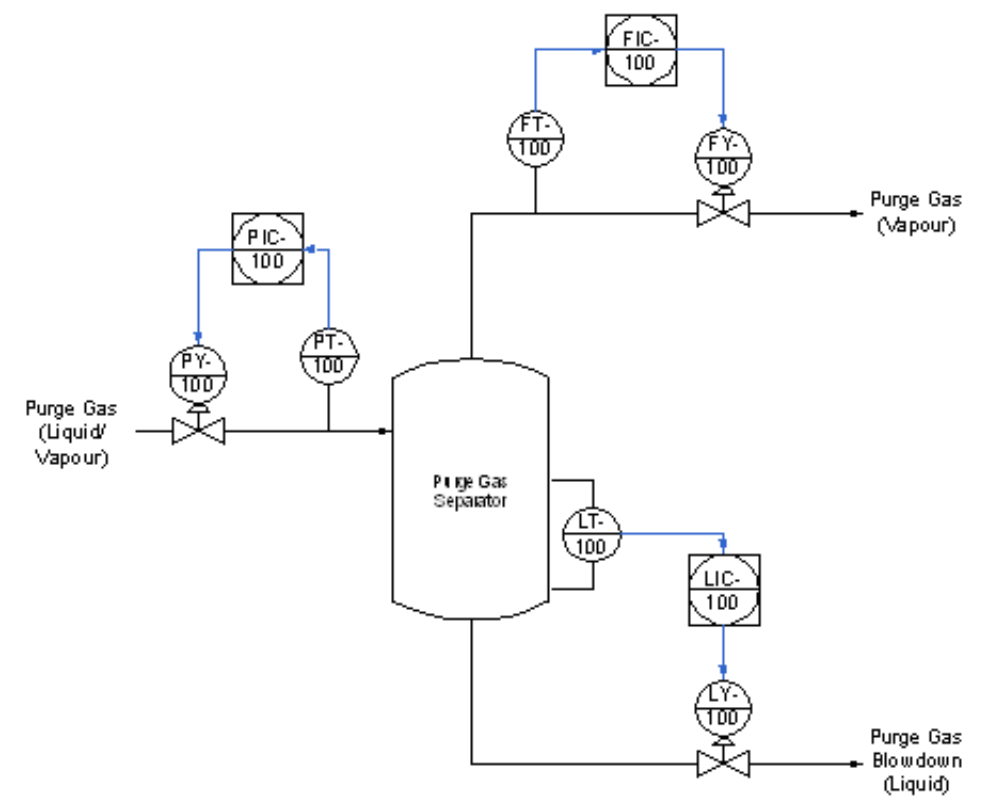

Figure 6: Separation Vessel Schematic 


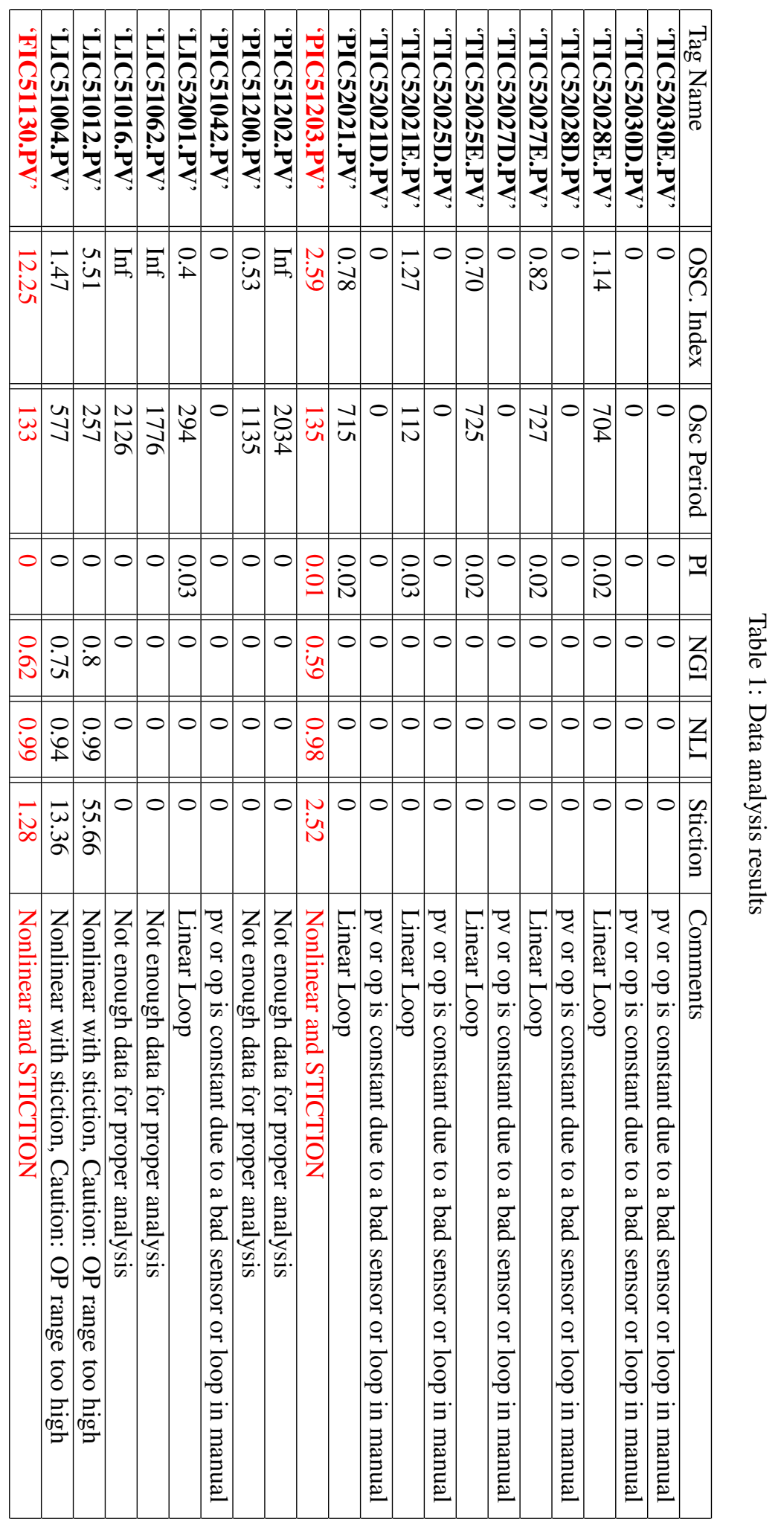


revealing the wide variation of process characteristics. The schematic process flow diagram of the unit is shown in Figure 5.

The unit has 21 control loops. Data were collected with a sampling period of $1 \mathrm{~s}$ for these loops. The data analysis results are shown in Table 1. The table shows that two control loops are suffering from valve stiction. When P\&I diagram of the process was consulted, it was found that they belong to the purge gas separator vessel. The schematic of the purge gas separator vessel is shown in Figure 6.

This unit of process includes three coupled control loops - input pressure control loop (PIC), Flow control loop (FIC) and the level control loop (LIC). Standard PI control has been used on all these loops. Normalized time trend data for these loops are shown in Figure 7. It can be observed from this figure that Loop PIC and FIC have sustained oscillation with one dominant frequency. This indicates that the oscillations in flow and pressure control loops may have been caused by one single source. Loop LIC shows that the controller output is saturated at $0 \%$ causing an oscillation with a period of 313 samples.

From Table 1, it can be observed that all three control loops are nonlinear. The $p v-o p$ plots for both flow and pressure control loops are shown in Figure 8. As can be seen from Figure 8, the automatic stiction detection algorithm finds stiction in both flow control loop and pressure control loops. This is the limitation of currently available stiction detection methods because all methods ignore the multivariate nature of the process industries. Since both loops had interactions, it is possible that only loop has valve stiction and the other is getting the oscillation from it. Therefore, another test is needed to find the actual sticky valve. The nonlinearity index $(N L I)$ suggests that the nonlinearity in loop FIC is higher than that of the PIC loop. On further testing of the flow loop only in manual mode it was found that movement of the flow loop valve in the 5-40\% range caused no change in the flowrate. Therefore it was concluded that the flow control valve has stiction problems. The positioner of this valve was replaced with a new positioner. Figure 9 shows the behaviour of the PV's before and after the positioner change. The figure is obtained from the DCS screenshot.

Figure 9 shows the PV of the loops before and after the positioner was changed. Before the problem was detected by stiction detection algorithm, plant engineers tried to resove the problem by tuning the controller. From the figure, it can be observed clearly that the loops performance could not be improved with just tuning before the positioner change (in the left). After the positioner was replaced, the oscillations were eliminated from the FIC and PIC loops. However, the level loop was still oscillating. A close look at the con- troller output data of this loop shows that this valve was saturating near $0 \%$ position. Retuning the level loop controller removes the saturation and the oscillation as well. Figure 10 shows the behaviour of all three loops after the positioner of the flow control valve of the FIC loop was changed and the level controller was retuned. It is clear from this figure that the whole unit is operating smoothly.

\section{Conclusion}

This paper described a higher order statistics based method to identify root-causes of poorly performing control loops. Two indices - NGI and NLI - can be used to identify nonlinear control loops in a plant. Then the specific pattern of the pv-op mapping of each control can be used to diagnose the problem further. The stabilization of an industrial process unit operation through the use of data driven techniques have been presented. The industrial case study demonstrates the practicality and usefulness of the technique described in this study.

\section{References}

[1] Choudhury MAAS, Shah SL and Thornhill NF, Diagnosis of Poor Control Loop Performance Using Higher Order Statistics, Automatica, 2004. 40(10):pp. 1719-1728

[2] Desborough L and Miller R, Increasing customer value of industrial control performance monitoring - Honeywell's experience, in AIChE Symposium Series, 326, 2002. pp. 153-186

[3] Desborough L and Harris T, Performance Assessment Measures for Univariate Feedforward / Feedback Control, The Canadian Journal of Chemical Engineering, 1992. 71:pp. 605616

[4] Harris TJ, Assessment of Control Loop Performance, The Canadian Journal of Chemical Engineering, 1989. 67:pp. 856861

[5] Stanfelj N, Marlin TE and MacGregor JF, Monitoring and Diagnosing Process Control Performance: The Single-Loop Case, Ind Eng Chem Res, 1993. 32:pp. 301-314

[6] Huang B and Shah S, Performance Assessment of Control Loops: Theory and Applications, Springer-Verlag, Germany, 1999

[7] Jelali M, An overview of control performance assessment technology and industrial applications, Control Engineering Practice, 2006. 14:pp. 441-466

[8] Luyben W and Hendershot DC, Dynamic Disadvantages of Intensification in Inherently Safer Process Design, Ind Eng Chem Res, 2004. 43:pp. 384-396

[9] Birgitta K, PID Controllers: Design and Evaluation, Ph.D. thesis, Chalmers University of Technology, Sweden, 2003

[10] Adeleye O, Design and Evaluation of optimal PID Controllers, Master's thesis, University of Alberta, Canada, 2006

[11] Choudhury MAAS, Shah SL and Thornhill NF, Diagnosis of Process Nonlinearities and Valve Stiction - Data Driven Approaches, Springer-Verlag, Germany, 2008

[12] Choudhury MAAS, Detection and Diagnosis of Control Loop Nonlinearities, Valve Stiction and Data Compression, $\mathrm{PhD}$ thesis, University of Alberta, Canada, 2004

[13] Hagglund T, A control loop performance monitor, Control Engineering Practice, 1995. 3(11):pp. 1543-1551

[14] Horch A, A Simple Method for Detection of Stiction in Control Valves, Control Engineering Practice, 1999. 7:pp. 1221-1231 

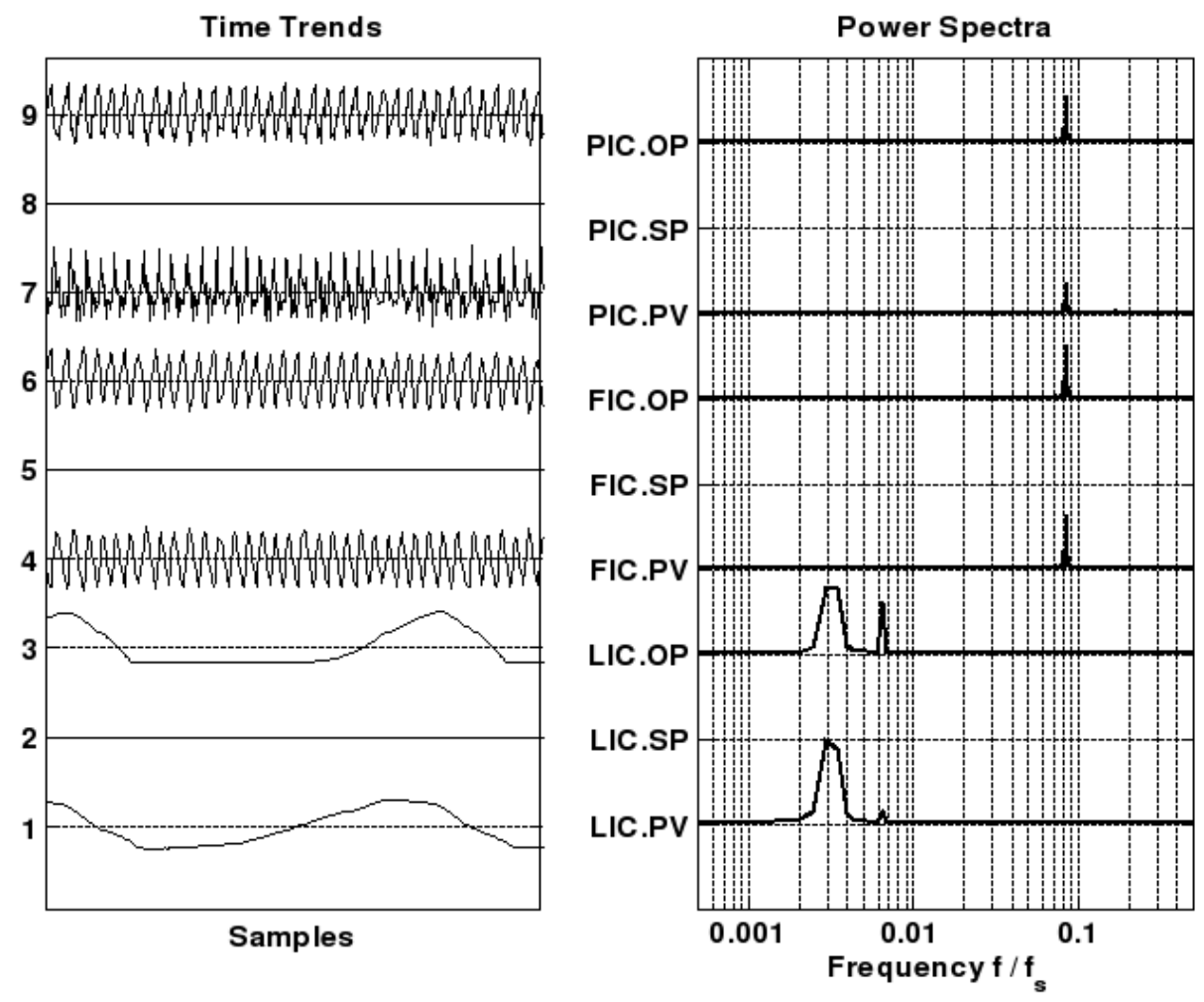

Figure 7: Time trend data of the separation vessel control loops

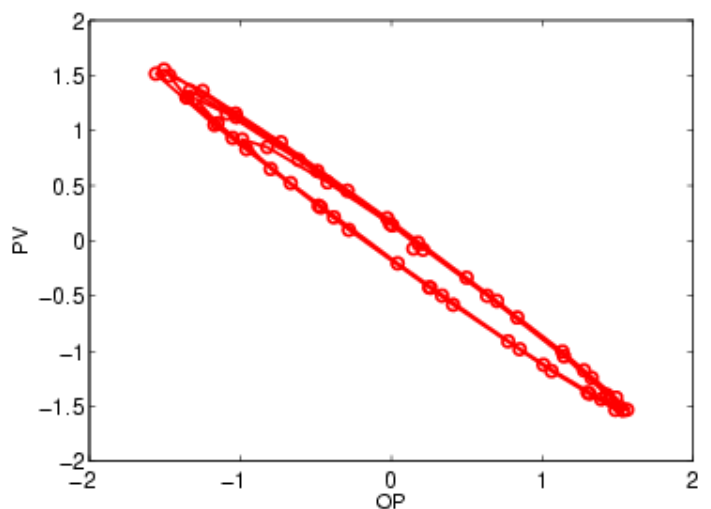

(a)

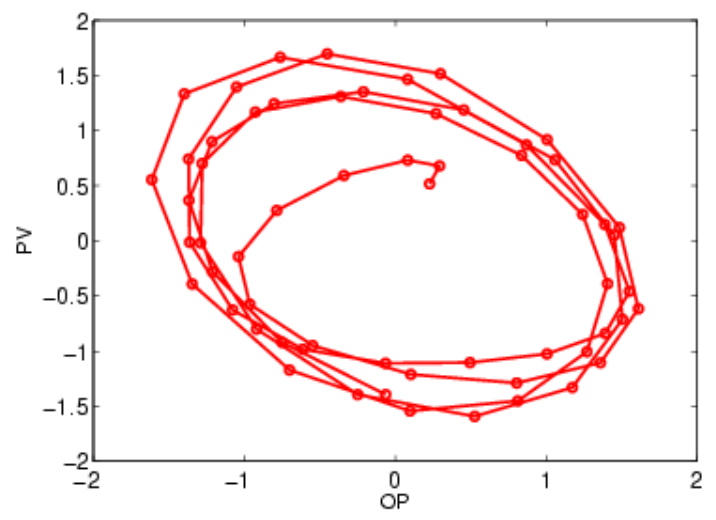

(b)

Figure 8: (a) PV-OP plot for FIC loop, (b) PV-OP plot for PIC loop 


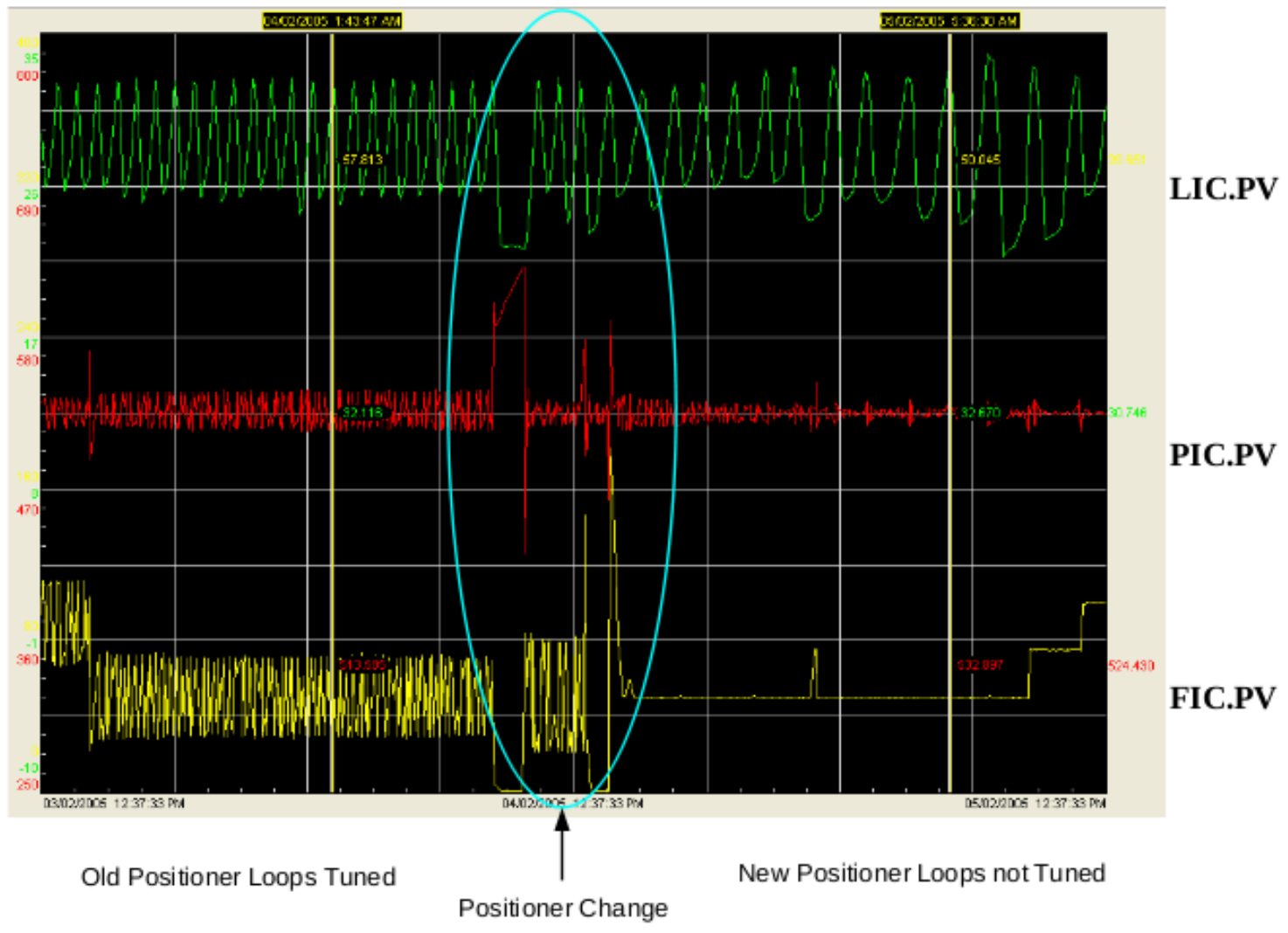

Figure 9: Control loop $p v$ 's before and after positioner change

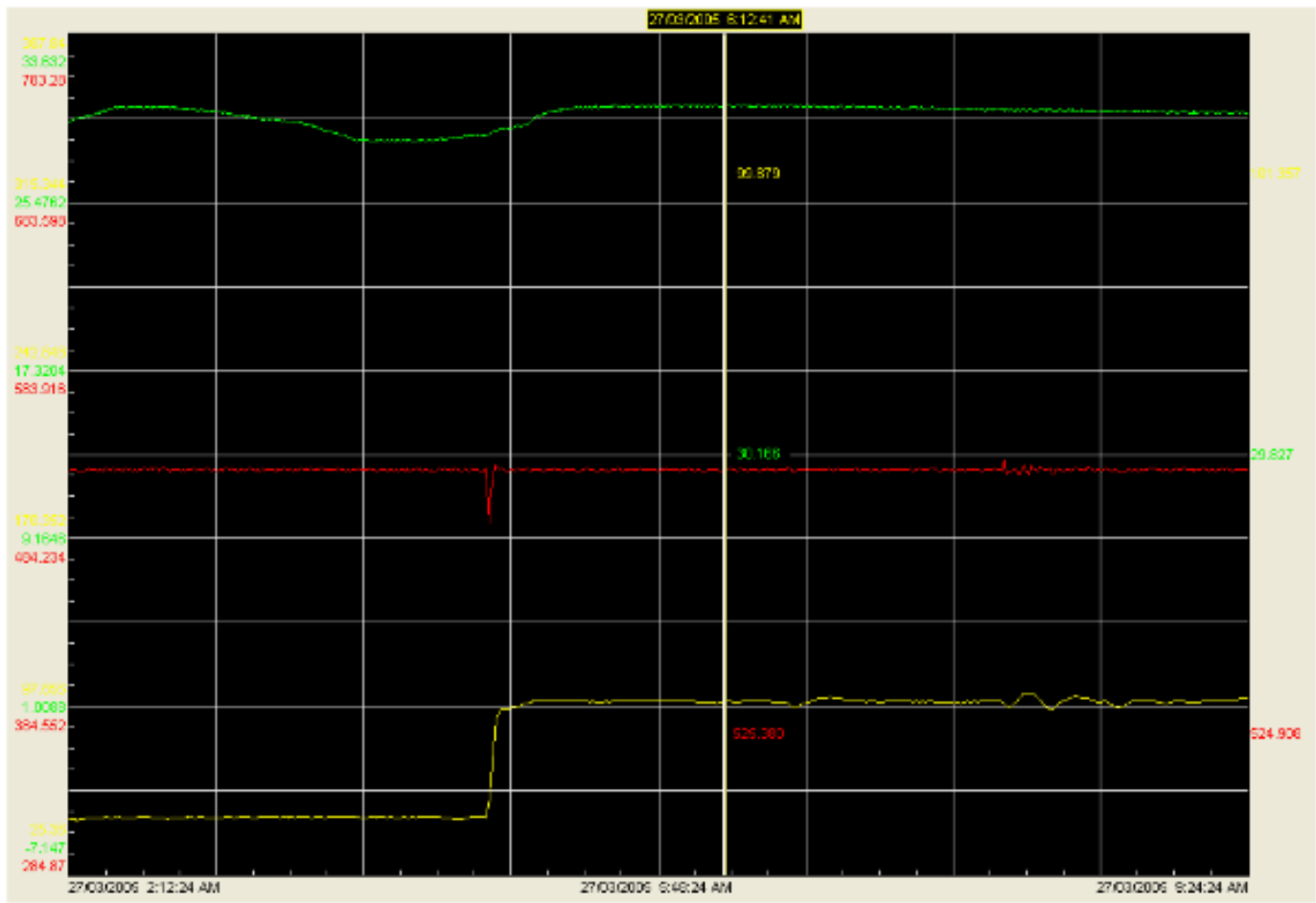

New Positioner Loops Tuned

Figure 10: Loop $p v$ 's after tuning with new positioner 
[15] Rengaswamy R, Hagglund $\mathrm{T}$ and Venkatasubramanian $\mathrm{V}, A$ qualitative shape analysis formalism for monitoring control loop performance., Engng Appl Artificial Intell, 2001. 14:pp. 23-33

[16] Ruel M, Stiction: The Hidden Menace, $\quad$ Control Magazine, 2000. Http://www.expertune.com/articles/RuelNov2000/stiction.html

[17] Choudhury MAAS, Thornhill NF and Shah SL, Modelling Valve Stiction, Control Engineering Practice, 2005. 13:pp. $641-658$ 